\title{
Uso da informática na atenção primária à saúde: Percepção dos enfermeiros
}

\author{
Use of information technology in primary health care: Nurses' perception \\ Uso de las tecnologías de la información en la atención primaria de salud: Percepción de las \\ enfermeiras
}

Recebido: 17/05/2021 | Revisado: 23/05/2021 | Aceito: 24/05/2021 | Publicado: 09/06/2021

\author{
Josiane Lima da Silva \\ ORCID: https://orcid.org/0000-0002-5219-5415 \\ Universidade Paranaense, Brasil \\ E-mail: josiane.188408@edu.unipar.br \\ Jolana Cristina Cavalheiri \\ ORCID: https://orcid.org/0000-0002-9549-8985 \\ Universidade Paranaense, Brasil \\ E-mail: Jolana_cc@hotmail.com
}

\begin{abstract}
Resumo
O presente estudo buscou identificar a percepção dos enfermeiros da atenção primária à saúde sobre o uso do computador e da informática em suas atividades diárias. Trata-se de uma pesquisa de campo, exploratória, descritiva, com abordagem qualitativa, por meio de aplicação de formulário via Google Docs, contendo questões norteadoras, confeccionadas pela pesquisadora, com base na literatura. A amostra foi de 19 enfermeiros com idade variando de 22 a 55 anos, em sua maioria do sexo feminino. Estes não possuíam curso específico em informática e consideravam o seu conhecimento como nível básico. Contudo, constatou-se que o computador e a informática auxiliavam na organização e planejamento do cuidado, permitindo a otimização do trabalho, tornando não só mais ágeis os atendimentos, como também mais qualificados, seguros e apropriados. Entretanto, essas ferramentas estavam limitadas por motivos técnicos e operacionais.
\end{abstract}

Palavras-chave: Sistemas de informação; Informática em saúde pública; Tecnologia da informação.

\begin{abstract}
This study aimed to identify nurses' perception of primary health care about the use of computers and information technology in their daily activities. It is a field research, exploratory, descriptive, with a qualitative approach, through the application of a form via Google Docs, containing guiding questions, made by the researcher, based on the literature. The sample consisted of 19 nurses with ages varying from 22 to 55 years old, mostly female. When asked if they had a specific computer course, most did not have it considering their knowledge as a basic level. However, it was found that the computer and information technology assist in the organization and planning of care, allowing the optimization of work, making not only the assistance more agile but also with greater quality, safety and resolution. However, these tools were limited for technical and operational reasons.
\end{abstract}

Keywords: Information systems; Public health informatics; Information technology.

\section{Resumen}

El presente estudio buscó identificar la percepción de las enfermeras sobre la atención primaria de salud sobre el uso de computadoras y tecnologías de la información en sus actividades diarias. Se trata de una investigación de campo, exploratoria, descriptiva, con enfoque cualitativo, mediante la aplicación de un formulario vía Google Docs, que contiene preguntas orientadoras, realizadas por el investigador, basadas en la literatura. La muestra estuvo constituida por 19 enfermeras con edades comprendidas entre los 22 y los 55 años, en su mayoría mujeres. Estos no tenían un curso específico de informática y consideraban sus conocimientos como un nivel básico. Sin embargo, se encontró que la informática y las tecnologías de la información ayudaron en la organización y planificación de la atención, permitiendo la optimización del trabajo, haciendo no solo la asistencia más ágil, sino también más calificada, segura y adecuada. Sin embargo, estas herramientas fueron limitadas por razones técnicas y operativas.

Palabras clave: Sistemas de información; Informática en salud pública; Tecnología de la información.

\section{Introdução}

A atenção Primaria à Saúde (APS) destaca-se como porta de entrada para o sistema público de saúde brasileiro e é responsável pelo atendimento de baixa complexidade em nível ambulatorial. A resolução de $80 \%$ dos casos atendidos está sob 
sua responsabilidade, e apenas quando necessário é realizado o encaminhamento a suportes de maior complexidade (Portela, 2017).

Diante dos serviços ofertados e reconhecendo a importância desse nível de atendimento, o Ministério da Saúde investiu em meios de melhorar seu funcionamento, e identificando as fragilidades existentes decidiu optar pela informatização, criando, por meio da Portaria n¹.412, de 10 de junho de 2013, o e-SUS Atenção Básica (e-SUS AB) com à implantação de dois sistemas de software: Coleta de Dados Simplificada (CDS) e Prontuário Eletrônico do Cidadão (PEC) (Cavalcante et al., 2016).

Com a implantação dos sistemas de informatização, o enfermeiro da APS passou a ser o principal agente de manipulação desses meios, fazendo uso dessa tecnologia para auxílio em suas decisões clínicas ou gerenciais (Jensen et al., 2016). Dessa forma, diante da grande demanda de lançamentos nos sistemas e manipulação do computador, vê-se a necessidade de capacitação dos trabalhadores. Além disso, por desenvolver ações de gerenciamento, o profissional deve compreender a necessidade de estar disponível às mudanças no processo de trabalho, incluindo a utilização de ferramentas tecnológicas, a capacitação da equipe quanto à utilização da tecnologia, bem como a avaliação dos processos desenvolvidos (Santos et al., 2017), e para que ocorra o funcionamento adequado dos serviços quanto ao uso da informatização, necessita-se melhorar os recursos disponíveis, suprindo a necessidade de computadores, treinamento e atualização periódica (Matsuda et al., 2015).

Tendo em vista o número expressivo de atendimentos e os investimentos em programas tecnológicos na Atenção Primária à Saúde, que geram novos desafios e a necessidade do manejo de computadores no gerenciamento efetivo da unidade, a pergunta principal deste estudo foi "Qual a percepção dos enfermeiros quanto ao uso do computador e da informática nas atividades realizadas na Atenção Primária à Saúde?” Neste contexto, o objetivo desta pesquisa foi identificar a percepção dos enfermeiros das Unidades Básicas de Saúde (UBS) referente ao uso da informática e do computador nas suas atividades diárias.

\section{Metodologia}

Trata-se de uma pesquisa de campo, exploratória, descritiva, com abordagem qualitativa, realizada com enfermeiros da atenção primária à saúde, por meio da aplicação de um formulário via Google Docs.

Os participantes do estudo foram os enfermeiros das unidades básicas de saúde, que aceitaram participar do estudo mediante a assinatura do Termo de Consentimento Livre e Esclarecido (TCLE), que foi disponibilizado de forma digital, sendo a amostragem por conveniência. Os critérios de inclusão foram todos os profissionais atuantes na atenção primária do município de estudo, que atuavam no serviço há pelo menos um mês, sendo exclusos aqueles que deixaram o questionário incompleto.

A coleta de dados ocorreu entre julho e agosto de 2020, após a aprovação do projeto pelo Comitê de Ética, e o consentimento para coleta de dados pela prefeitura. Para iniciar a coleta das informações foi solicitado à Secretaria Municipal de Saúde o e-mail dos enfermeiros que atuavam nos serviços. Em seguida foi disparado o formulário, acompanhado das orientações sobre a pesquisa a todos os e-mails disponibilizados. Entretanto, devido à pouca participação, após passar algumas semanas foi entrado em contato com o participante via telefone para confirmar o recebimento do formulário, indagar sobre a participação e esclarecer eventuais dúvidas, e então foi reenviando o TCLE e o formulário para preenchimento.

O formulário via Google Docs foi confeccionado conforme a literatura brasileira sobre a temática e possuía as seguintes variáveis: idade, sexo, cor, estado civil, religião, nível de formação e tempo de atuação. Posteriormente os questionamentos foram realizados por questões abertas, como: Quais os Softwares/Programas que você utiliza na sua unidade 
de saúde? Como a informatização tem auxiliado no seu trabalho como enfermeiro? Fale da importância do uso do computador para o cuidado de enfermagem? Quais as dificuldades e pontos de sucesso encontrados por você, para uso da informática e do computador em seu trabalho? Você tem dificuldade com o uso do computador/Informática? Em quais aspectos? O que você sugere para melhorar o uso do computador e da Informatização na Atenção Primária?

O processo de análise de dados ocorreu em setembro de 2020. As questões da entrevista foram transcritas na íntegra e em seguida submetidas à organização de conteúdo, por meio do Método de Bardin, ou seja, classificando as informações em categorias e subcategorias. Os participantes foram identificados pela palavra "Enfermeiro" em ordem crescente de participação. Inicialmente foram apresentados os resultados expondo as falas dos participantes, posteriormente discutido as categorias em capítulos.

O projeto foi encaminhado ao Comitê de Ética em Pesquisa com Seres Humanos da Universidade Paranaense (Unipar), seguindo as recomendações éticas em pesquisa de acordo com a Resolução no 466/2012 do Conselho Nacional de Saúde e aprovado sob o Parecer $n^{\circ} 4.053 .715$.

\section{Resultados}

Participaram do estudo 19 enfermeiros com idade variando de 22 a 55 anos, sendo $84 \%$ do sexo feminino e brancos, seguido de $16 \%$ homens e pardos, em relação ao estado civil 58\% eram casados. A religião predominante foi a católica com 79\%; 42\% eram pós-graduados, com tempo de atuação na enfermagem de mais de dez anos e tempo de trabalho na unidade inferior a cinco anos.

Quando questionados se possuíam algum curso especifico na área de informática 74\% negaram, dos participantes $68 \%$ consideraram o seu conhecimento como nível básico. Os enfermeiros que relataram possuir algum curso especifico se referiram a capacitações do pacote do Microsoft Office.

Quando realizada a análise dos discursos dos participantes, obtiveram-se as seguintes categorias: "Tecnologia como ferramenta do planejamento e longitudinalidade do cuidado", "Compartilhamento de informações através da informática", "Ferramentas e programas utilizados no gerenciamento do cuidado", "Aspectos negativos e positivos da informatização" e "Sugerindo melhorias".

\section{Tecnologia como ferramenta do planejamento e longitudinalidade do cuidado}

Especialmente quanto ao uso do computador e da informática para o cuidado de enfermagem, os enfermeiros relataram a possibilidade de planejamento e gerenciamento do cuidado, bem como a longitudinalidade da oferta da assistência, caracterizando a categoria, conforme os discursos abaixo:

\footnotetext{
“Contribui para o cuidado sistematizado, compartilhamento de informações na rede” (Enfermeiro 8).

"De suma importância para unificação dos dados e planejamento da assistência de maneira integral" (Enfermeiro 13).

"Através de sistemas e programas a equipe consegue organizar e gerenciar o tempo de atendimento, facilitando assim a organização e a rotina de unidade. Com os registros e relatórios é possível acompanhar a evolução do paciente [...]" (Enfermeiro 14).
} 


\section{Compartilhamento de informações através da informática}

Os enfermeiros foram questionados sobre como a informatização tem auxiliado em seu trabalho, e evidenciaram que otimiza a organização das atividades, melhora a comunicação, o compartilhamento de informações e a qualidade dos atendimentos, conforme pode-se observar nas falas a seguir.

"Registra informações de forma precisa e segura, organização do trabalho, legibilidade nos prontuários, facilita pesquisas e estudos." (Enfermeiro 1)

“O maior ganho creio que seja o compartilhamento das informações.” (Enfermeiro 2)

"Facilita o registro e melhora a qualidade do atendimento. Permite ter acesso ao prontuário eletrônico interligado aos demais serviços do município, contribuindo para o atendimento prestado, com relação ao manejo dos casos e tomada de condutas, ações." (Enfermeiro 6).

"Organização e maior confiabilidade das informações. Facilidade de acesso a todas as informações do paciente, desde dados pessoais a consultas e procedimentos realizados, e medicamentos em uso.” (Enfermeiro 14).

\section{Ferramentas e Programas utilizados no gerenciamento do cuidado}

Nesta perspectiva, os programas e softwares aludidos pelos enfermeiros no gerenciamento do cuidado e do serviço, estavam os pacotes básicos do Word, os programas do Ministério da Saúde e o WinSaude, conforme as falas a seguir.

“Word, Excel, WinSaude.” (Enfermeiro 1).

“Ids Saúde e páginas da web do Ministério da Saúde.” (Enfermeiro 2).

"Software básico: Word, Excel, etc. e programas como os do Ministério da Saúde: CadSUS Web, Siscan, etc." (Enfermeiro 4).

“Word, WinSaude.” (Enfermeiro 12).

\section{Aspectos negativos e positivos da informatização}

Sobre quais as dificuldades e pontos de sucesso quanto ao uso da informática e computador, desprenderam-se duas subcategorias das falas dos participantes. A primeira faz alusão às dificuldades encontradas, dentre as quais citaram-se: $o$ uso de computadores e tecnologias ultrapassadas, queda na internet, falta de capacitação e dificuldade apresentada pelos profissionais mais antigos para efetuar o registro. Além disso, dois profissionais referiram a ausência de privacidade e sigilo das informações contidas no prontuário e a diminuição do contato com o paciente, conforme segue.

“[...] profissionais mais antigos recusam-se a registrar as informações eletronicamente [...]” (Enfermeiro 1).

“Dificuldades: Falta de treinamento e dificuldade de acesso à internet, eventualmente [...]” (Enfermeiro 6).

“Dificulta quando o computador ou a internet não funciona [...]” (Enfermeiro 10).

"Alguns colegas não usam como instrumento de trabalho, mas sim com coisas pessoais e desnecessárias." (Enfermeiro 3).

Quanto aos pontos de sucesso, os profissionais referiram a economia de tempo e recurso, especialmente a diminuição do uso de papel para registro, a busca rápida de informações, a facilidade de seguimento dos pacientes, bem como a diminuição dos erros de prescrição, como se destaca a seguir. 
"Economiza tempo e recursos, possibilita a visualização das informações registradas por toda a equipe multiprofissional." (Enfermeiro 1).

“[...] acesso rápido de informações que contribuem no atendimento ao paciente.” (Enfermeiro 6).

“[...] agilidade, fidedignidade nos dados, diminuição de erros nas prescrições.” (Enfermeiro 9).

“[...] sem documentos de papeis agiliza muito o trabalho.” (Enfermeiro 17).

“[...] seguimento de todos.” (Enfermeiro 7).

\section{Sugerindo melhorias}

Para contextualizar às sugestões dos profissionais, quanto ao uso do computador na atenção primária, citaram-se: necessidade de cursos de aperfeiçoamento; melhoramento da estrutura da rede, equipamentos e internet das unidades; e melhora dos registros por parte dos profissionais, segundo as falas adiante.

"Solucionar problemas como a sobrecarga do sistema e queda de internet." (Enfermeiro 1).

“Cursos de aperfeiçoamento.” (Enfermeiro 5).

“Politica de Estado com financiamento de estrutura de rede, equipamentos, capacitação.” (Enfermeiro 8).

“Acredito que para o momento atual melhorias na rede ajudariam a evitar de ficar sem ter a acesso ao prontuário do paciente uma vez que necessitamos estar on-line, [...] capacitações para melhor uso de alguns recursos que os programas nos oferecem." (Enfermeiro 9).

\section{Discussão}

Este estudo possibilitou uma reflexão sobre o uso da informática na atenção primária à saúde, demonstrando a necessidade de mudanças nos serviços para os profissionais desenvolverem seus registros de forma adequada. Referente às limitações encontradas nessa pesquisa, destacaram-se a coleta de dados realizada por meio de formulário via Google Docs, a não adesão de todos os profissionais e o número limitado de pesquisa sobre a temática, sendo necessário aprofundamento em novos estudos para completar as lacunas.

Quanto ao perfil dos enfermeiros observou-se a prevalência de mulheres, com mais de 20 anos, cor branca, casadas, católicas, com especialização e experiência na profissão e atenção primária, o que vai ao encontro de estudo desenvolvido em capital do Sul do Brasil, o qual também obteve a presença do sexo feminino, com idade superior a 30 anos e tempo de atuação de até 20 anos (Gonçalves et al., 2016). Da mesma forma, estudo desenvolvido no Ceará obteve maior parte da amostra do sexo feminino, casadas, com religião católica, possuindo títulos de pós-graduação, mestrado e doutorado, com tempo de atuação na área maior que 10 anos (Araújo et al., 2019).

A maioria dos profissionais referiu ter nível básico em relação ao conhecimento que possuía quanto a informatização e o uso de computadores. Esse fato assemelha-se aos apresentados em outra pesquisa, na qual os participantes relataram ser iniciantes no domínio da informática, mesmo utilizando o computador em sua rotina diária (Gonçalves et al., 2015). Quanto à presença de cursos nesta área, grande parte relatou não os ter e quando possuía era a capacitação básica, o que vai ao encontro de estudo realizado na região oeste de Minas Gerais, que reconheceu a falta de conhecimento dos profissionais sobre como operacionalizar os sistemas para extrair informações, atrelada às mudanças súbitas e constantes nos instrumentos tecnológicos (Cavalcante et al., 2018). 
Em relação ao o uso da informática e do computador para planejamento da assistência de enfermagem, bem como da organização dos serviços, a informatização pode aumentar a segurança do paciente, sendo que a função mais utilizada diz respeito ao monitoramento de desempenho dos serviços e planejamento das unidades de saúde (Santos et al., 2017).

Pesquisa feita em uma UBS ressaltou que todos os profissionais utilizavam sistemas informatizados nas atividades, o que agilizava a rotina, contribuía para melhora da assistência e comunicação entre a equipe, e, além disso, o PEC pôde ser utilizado como ferramenta para planejamento e gerenciamento, por meio dos relatórios fornecidos pelo sistema (Martins et al., 2017). Esses dados são condizentes com as falas dos enfermeiros, quando ressaltam a unificação dos dados pelo uso do PEC, bem como o planejamento da assistência, a melhoria na organização do tempo, das rotinas dos serviços e dos registros.

Ainda nesta categoria observou-se que os profissionais aludiram o uso da informática para longitudinalidade do cuidado. Esta consiste na assistência ao usuário por um longo período de tempo, ou seja, um vínculo de longa duração entre os profissionais de saúde e os pacientes (Baratieri \& Marcon, 2011). Diante disso, percebeu-se nos discursos dos profissionais que o uso de sistemas informatizados auxilia na oferta de um cuidado sistematizado e integral, o que concorda com pesquisa desenvolvida com 12 enfermeiros, os quais ressaltaram que o computador facilita o acompanhamento do paciente, possibilita acesso instantâneo às informações, agiliza o cuidado e as decisões clínicas, sem a necessidade de deslocamento do setor, proporcionando maior tempo dedicado ao cliente (Matsuda et al., 2015).

Ainda, estudo desenvolvido no Rio Grande do Sul ressalta que a tecnologia proporciona melhor acompanhamento a grupos prioritários, segurança nos registros do paciente e comunicação rápida e facilitada entre a equipe, assim como melhoria e organização no processo de trabalho (Pissaia et al., 2016). Ademais, outra pesquisa com enfermeiros ressaltou que o uso do computador possibilita melhor administração do tempo, facilita vislumbrar a real necessidade da sua população, predispor maior destreza para exercício da consulta de enfermagem, constituindo vínculo e confiança com o cliente e estabelecendo uma relação satisfatória (Ferreira et al., 2018), assemelhando-se aos discursos apresentados.

Igualmente se observou pelas falas dos profissionais que o compartilhamento de informações está presente. Estudo com a mesma temática, realizado em Pernambuco, referiu que a junção de dados descomplica e acelera o atendimento, reduzindo o tempo para a coleta e as informações a serem colhidas; além disso, o programa e-SUS AB proporciona uma forma facilitada de realizar o trabalho, especialmente no que se refere à moderação da burocracia, proporcionando aos profissionais uma avaliação sistemática e integral do estado de saúde do usuário (Medeiros et al., 2017).

Mediante o exposto, dados coletados em Minas Gerais apontam que a modernização desenvolve a automação dos processos e contribui para o cuidado, predispõe relatórios fidedignos para apoiar a gestão, propicia o despacho assertivo de medicação (se necessário), realiza a restauração dos registros clínicos e ainda promove armazenamento de referências sobre as famílias e comunidades (Cavalcante et al., 2018), o que pode ser compartilhado pelos diferentes profissionais que realizam a assistência em saúde.

Por conseguinte, pesquisa realizada no Sul do Brasil relata o PEC como um acervo informatizado do estado de saúde dos cidadãos, que dispensa registro em papel, é acondicionado e conduzido em inteira confidencialidade - pois é acessível apenas ao usuário autorizado por intermédio de login e senha - ainda possibilita a organização dos dados automaticamente por tipos de atendimentos e datas, viabilizando acessos retrospectivos de maneira ágil (Martins et al., 2017). Essa caracterização está de acordo com os discursos dos profissionais que aludiram a legibilidade dos registros nos sistemas, a confidencialidade dos dados, a interligação entre os serviços e a melhora da qualidade da assistência.

Os enfermeiros do presente estudo referenciaram que os sistemas informatizados proporcionam o acompanhamento do paciente pelos diferentes serviços, de forma semelhante ao relatado em uma pesquisa realizada em São Paulo, a qual refere que o uso do PEC permite aos profissionais realizar o mapeamento do aglomerado de informações, oportuniza avaliação da cobertura e as metas atingidas, promove uma padronização na coleta de dados e direciona as ações em saúde com base em 
históricos reais, garantindo um melhor gerenciamento de intervenções, impulsionando mudanças positivas e o compartilhamento de informações sobre o estado de saúde do paciente com diferentes profissionais, bem como em serviços diferenciados da rede de atenção à saúde (Ferreira et al., 2018).

Quanto aos programas e softwares mencionados pelos enfermeiros como ferramentas utilizadas no planejamento do cuidado e no gerenciamento da unidade, observou-se referência aos pacotes básicos do Word. Acredita-se que os profissionais aludiram à utilização dos pacotes do Office, entre eles o Word, por ser um dispositivo de escrita com possibilidade de edição, ou seja, um processador de texto que permite atualizar informações conforme a necessidade. Além disso, por ser o programa instalado e disponibilizado nos computadores ofertados pelo município, verificou-se que os profissionais utilizavam-no, corriqueiramente, para organização dos serviços disponibilizados à população e para o planejamento dos recursos humanos e físicos dos serviços. Pesquisa realizada no Sul do Brasil relatou utilizar o computador de maneira semelhante, incluindo o uso de processadores de texto para desenvolvimento de relatórios, seguido de correio eletrônico e pesquisa de referências bibliográficas que fortalecem o cuidado ao paciente (Gonçalves et al., 2016).

Por conseguinte, dentre os programas do Ministério da Saúde relatados na fala dos enfermeiros está o Siscan, ferramenta virtual que integra os Sistemas de Informação do Câncer do Colo do Útero (Siscolo) e do Câncer de Mama (Sismama). Essa aplicabilidade proporciona aos profissionais e às Unidades de Saúde acesso a exames já solicitados e inclusão de novos, assim como informações de procedimentos relacionados à detecção precoce e à confirmação diagnóstica dessas neoplasias. Este é Integrado ao Cadastro Nacional de Cartão Saúde (CadSUS) (Brasil, 2013), demonstrando a assistência dos profissionais à saúde das mulheres.

Outro programa citado foi o Sistema de Controle Logístico de Insumos Laboratoriais (Sislog), local virtual onde se realiza pedido de testes rápidos, acessado através de login e senha, por profissional cadastrado (Brasil, 2016). Em continuidade às falas dos entrevistados, contatou-se também o acesso ao Sistema de Informações do Programa Nacional de Imunizações (PNI), utilizado pelos profissionais atuantes nas salas de vacinas, que auxilia na gestão dos atendimentos, do acompanhamento de estoque e da distribuição dos imunobiológicos (Brasil, 2020).

Os sistemas informatizados manuseados nas unidades de saúde são diversos, alguns disponibilizados pelo Ministério da Saúde e outros pelo município, são mecanismos padronizados de monitoramento que fornecem dados essenciais para direcionar ações de interesse público, para a geração de dados epidemiológicos, e seu manuseio fica a critério da equipe da unidade, sendo alguns exclusivos do enfermeiro (Daniel et al., 2014).

Em todas as falas dos profissionais observou-se a referência ao programa informatizado WinSaude, também conhecido e citado como IDS SAÚDE. Trata-se de uma ferramenta de gestão municipal que fornece a junção de todos os dados da localidade, disponível aos diferentes setores de saúde do município e interligado aos sistemas do Datasus, do Ministério da Saúde. Incluso nesse sistema - e de suma importância - está o PEC, que fornece acesso ao banco de dados, com integralidade das informações do cliente, e proporciona a gerência do fluxo de atendimento (Dengo, 2020).

Acredita-se que os profissionais tenham aludido o uso desta ferramenta por ser um software disponibilizado pela Secretaria de Saúde, com objetivo de melhorar os serviços oferecidos, sendo contratado conforme as necessidades do cliente. Estudo realizado em uma capital do Sul do Brasil identificou também o manuseio de processador de texto específico e maior acesso ao sistema do PEC para registro de anotações e avaliações de enfermagem (Gonçalves et al., 2016). O PEC proporciona dados completos, individualizados, integrados e possibilita eliminar fichas de preenchimento manual, abolindo o retrabalho e facilitando o processo de coleta de informações; da mesma forma, sempre está legível e é mais confiável (Cavalcante et al., 2018).

Sobre as dificuldades e pontos de sucesso quanto ao uso da informática e do computador, observou-se como fator negativo o fato de os profissionais mais antigos recusarem-se a utilizar os sistemas eletrônicos, o que vai ao encontro de estudo 
do interior paranaense com temática semelhante que revelou a dificuldade de manuseio da ferramenta por profissionais mais velhos (Matsuda et al., 2015).

Sabe-se que no cenário atual do mercado de trabalho destaca-se o encontro de gerações desafiando os gestores a administrar essas diferentes faixas etárias existentes dentro de uma unidade, enquanto a geração $\mathrm{X}$ faz parte do início da televisão, demonstram certa resistência às inovações e insegurança, a geração $\mathrm{Y}$, nasceu na era da tecnologia com recursos ilimitados e destacam-se em realizar multitarefas (Junior et al., 2016).

Além disso, os profissionais referiram falta de treinamento e capacitações, assim como em estudo efetuado em Queimadas-PB, o qual reconheceu que os treinamentos eram realizados de forma superficial na instalação do programa, o que dificultava o aprendizado eficiente, expunha a urgência de capacitações periódicas - principalmente após atualizações do sistema - e a carência de devolutivas dos gestores. Dessa forma, se faz necessária uma abordagem mais aprofundada, em especial nos lançamentos de dados, para evitar erros durante os registros (Medeiros et al., 2017). A divulgação e difusão eficiente de informações sobre os sistemas a serem utilizados faz com que os usuários os aceitem mais facilmente e adotemnos no processo de informatização (Cavalcante et al., 2018).

Observou-se ainda que os enfermeiros aludiram as dificuldades com máquinas antigas e com computadores, o que condiz com análise realizada em Minas Gerias, que ressaltou estrutura física inadequada e resistência dos profissionais às mudanças, ocasionando dificuldades de manuseio e baixa qualidade dos dados (Cavalcante et al., 2018). Além disso, um estudo de revisão integrativa ressaltou a insuficiência de recursos financeiros e humanos, o que agrega desmotivação profissional (Santos et al., 2017).

Não menos importante, observou-se a referência dos profissionais sobre a ausência de privacidade e sigilo das informações contidas no PEC. Segundo o Código de Ética da categoria, é dever da enfermagem prestar assistência sem qualquer discriminação, incentivando hábitos saudáveis à pessoa e à família; respeitar o pudor e garantir o sigilo e a intimidade da pessoa durante todo o seu atendimento, com absoluto anonimato das informações coletadas ou resultados obtidos (COFEN, 2017). Constata-se que existe a necessidade de educação continuada que enfoque além dos aspectos técnicos do uso das ferramentas, a importância de um atendimento ético, profissional, garantindo a confidencialidade e sigilo das informações, utilizando os sistemas para compartilhamento de informações referentes ao cuidado.

Quanto aos aspectos positivos, os profissionais referiram a economia de tempo e recurso, especialmente a diminuição do uso de papel para registro, a busca rápida de informações, a facilidade de seguimento dos pacientes, bem como a diminuição dos erros de prescrição. Os mesmos aspectos foram observados em outra pesquisa que apontou que o uso do computador proporcionou melhor gerenciamento de dados, registros sem uso de papel armazenado, facilidade de acessos e compartilhamento de dados de forma eficaz, o que fundamentou decisões e ajudou na elaboração de estratégias assertivas, contribuindo para a distinção das informações de usuários e das atividades profissionais (Cavalcante et al., 2019).

São muitos os pontos positivos das ferramentas de tecnologias de informação e comunicação, entre eles citam-se: substituição do prontuário de papel - o que é benéfico ao meio ambiente; melhoramento da qualidade da assistência, agregando maior rapidez nas decisões clínicas e maior segurança das informações relacionadas aos meios de acessos; melhoria do cuidado, devido à maior velocidade de resolução dos assuntos burocráticos, restando mais tempo para o cuidado; extinção da necessidade de deslocamento dos setores, pois as informações ficam organizadas automaticamente, possibilitando um acesso integral aos dados clínicos do paciente por vários setores (Cardoso et al., 2017).

Ressalta-se que os profissionais aludiram a diminuição dos erros de medicação relacionados à ilegibilidade da prescrição médica escrita, considerada um fator agravante e de risco à segurança do cliente. A prescrição por sistema informatizado, implantação da dose unitária e do código de barras na administração de medicamentos são fatores que contribuem para a redução dessa falha (Mieiro et al., 2019). 
Em relação às sugestões dos profissionais quanto ao uso do computador na atenção primária à saúde, os quais enfatizaram a necessidade de recursos para melhoria da rede de serviços, estrutura e capacitação. Similarmente, em outro estudo os profissionais mencionaram a necessidade de melhorias no programa, reclamaram do tempo gasto para realizar o processo de enfermagem, da lentidão do sistema e da necessidade de frequentes atualizações; notou-se a ânsia por uma maior disponibilidade de computadores potentes para abrir páginas e arquivos mais rapidamente (Juliani et al., 2014). Ressalta-se que apesar das vantagens de utilizar a informatização, ela traz consigo custos e que o serviço público precisa estar atento à necessidade de melhorar os serviços disponibilizados, para que haja melhoria na assistência.

Também se observou a referência à necessidade de capacitações e cursos de aperfeiçoamento, nas falas dos enfermeiros. Os softwares e as tecnologias em saúde são tendências aos serviços de saúde, sendo necessário aperfeiçoamento constante sobre o uso desses recursos (Assis \& Ramos, 2017).

A educação continuada em tecnologia se faz necessária, já que, se o profissional possuir domínio sobre a ferramenta, fará lançamentos de forma ágil, resultando em otimização do processo de trabalho e maior tempo destinado a assistência. Além disso, a instituição deve valorizar o desenvolvimento profissional, gerando menor estresse e aumentando a eficiência, a assertividade e o desempenho profissional, minimizando erros (Cardoso et al., 2014). Estudo semelhante também referiu como sugestão de melhorias o treinamento e o aperfeiçoamento, a maior disponibilidade de computadores, inclusive aquisição de dispositivos móveis, atualizações de softwares, assim como a necessidade de cadeiras mais ergonômicas (Matsuda et al., 2015).

Por fim, observou-se a necessidade de melhorias nos programas e softwares utilizados pelos profissionais, os quais destacaram a necessidade de estarem on-line para complementarem as informações. A conexão com a internet é fator primordial para conseguir acessar os programas nas unidades, gerar protocolos e envios de dados ao Ministério da Saúde, portanto, apesar de todas as unidade pesquisadas terem acesso a essa ferramenta, há descontentamento devido a quedas constantes e impossibilidade de acesso ao sistema informatizado, o que gera acúmulo de tarefas para posterior lançamento, sem mencionar a incapacidade de acesso a informações completas do paciente a qualquer momento.

\section{Considerações Finais}

O uso do computador faz parte da rotina dos serviços e dos profissionais enfermeiros, sendo utilizado na organização e no planejamento do cuidado, permitindo a otimização do trabalho, tornando não só mais ágeis os atendimentos, como também com maior qualidade, segurança e resolutividade, entretanto, está limitado a alguns motivos técnicos e operacionais.

Os profissionais apresentaram satisfação no uso de ferramentas e softwares no trabalho, salientando a economia de tempo, a diminuição dos erros de prescrição, a longitudinalidade do cuidado e relatórios e informações mais assertivos. Contudo, são necessários investimentos para ampliação e aprimoramento de infraestrutura e equipamentos, assim como treinamentos, capacitações e conscientização dos profissionais para o uso.

\section{Referências}

Araujo J. R., Filho D. C. A., Machado L. D. S., Martins R. M. G., \& Cruz R. S. B. L. C. C. (2019). Sistema e-SUS AB: percepções dos enfermeiros da Estratégia Saúde da Família. Saúde Debate. Rio de Janeiro. 43(122): 780-92. http://dx.doi.org/10.1590/0103-1104201912210

Assis M. A. \& Ramos J. M., (2017). Conhecimento dos graduandos de Enfermagem sobre o uso de Tecnologias Informatizadas. J. Health Inform. 9(2): 39-43. http://www.jhi-sbis.saude.ws/ojs-jhi/index.php/jhi-sbis/article/view/442.

Baratieri T. \& Marcon S.S., (2011) Longitudinalidade do Cuidado: Compreensão dos enfermeiros que atuam na Estratégia Saúde da Família. Esc Anna Nery. 15(4):802-10. https://doi.org/10.1590/S1414-81452011000400020.

Cardoso R. B., Ferreira B. J., Martins W. A. \& Paludeto S. B. (2017). Programa de educação permanente para o uso do prontuário eletrônico do paciente na enfermagem. J. Health Inform. 9(1):25-30. http://www.jhi-sbis.saude.ws/ojs-jhi/index.php/jhi-sbis/article/view/429/302. 
Cavalcante R. B., Esteves C. J. S., Gontijo T. L., Guimarães E. A. A., Machado R. M. \& Oliveira C. V. (2018). Informatização da Atenção Básica a Saúde: Avanços e Desafios. Cogitare Enfermagem. 2018 Ago; 23(3): e54297. http://dx.doi.org/10.5380/ce.v23i3.54297

Cavalcante R. B., Esteves C. J. S., Gontijo T. L., Brito M. J. M., Guimarães E. A. A. \& Barbosa S. P. (2019). Informatização da atenção primária à saúde no Brasil: a rede de atores. Rev. Bras. Enferm. Brasília. 2019 Abr; 72(2): 337-44. https://doi.org/10.1590/interface.180364

Conselho Federal de Enfermagem (Cofen). (2017). Resolução n ${ }^{\circ}$ 564/2017, de 06 de novembro de 2017. Dispõe sobre o Código de Ética dos profissionais de Enfermagem. Brasília. http://www.cofen.gov.br/resolucao-cofen-no-5642017_59145.html

Daniel V. M., Pereira G. V. \& Macadar M. A. (2014) Perspectiva Institucional dos sistemas de informação em saúde em dois estados brasileiros. RAC. 18(5):650-69. https://doi.org/10.1590/1982-7849rac20141709.

Dengo MC. (2020). IDS Saúde: O sistema para sua gestão de Secretaria de Saúde ser rápida e eficaz. Pato Branco. https://www.ids.inf.br/ids-saude/.

Ferreira S. R. S., Perico L. A. D. \& Dias V. R. F. G. A (2018). A complexidade do trabalho do enfermeiro na Atenção Primária à Saúde. Rev. Bras. Enferm. Brasília. 71(1): 704-709. http://dx.doi.org/10.1590/0034-7167-2017-0471

Gonçalves L. S., Castro T. C. \& Fialek S. A. (2015). Experiência Computacional de Enfermeiros Brasileiros de um Hospital Universitário. J. Health Inform. $2015 \mathrm{Jul} / \mathrm{Set} ;$ 7(3): 82-7. http://www.jhi-sbis.saude.ws/ojs-jhi/index.php/jhi-sbis/article/view/342.

Gonçalves L. S., Fialek S. A., Castro T.C. \& Wolff L. D. G. (2016). Experiência de Enfermeiros com Computadores na Atenção Primária: Estudo Exploratório. Rev Cogitare Enferm. 21(1): 1-11. http://ojs.c3sl.ufpr.br/ojs2/index.php/cogitare

Jensen R., Guedes E.S. \& Leite M. M. J. (2016). Competências em Informática Essenciais à Tomada de Decisão no Gerenciamento em Enfermagem. Rev Esc Enferm USP. 50(1): 112-20. https://doi.org/10.1590/S0080-623420160000100015

Juliani C. M. C. M., Silva M. C. \& Bueno G. H. (2014). Avanços da Informática em Enfermagem no Brasil: Revisão Integrativa. J. Health Inform. 2014 Out/Dez; 6(4):161-5. http://www.jhi-sbis.saude.ws/ojs-jhi/index.php/jhi-sbis/article/view/322.

Júnior C. C. M. C., Lima F. A., Conceção I. A., Souza W. A. \& Konrad M. A. (2016). O Gerenciamento das relações entre as múltiplas gerações no mercado de trabalho. Revista da Faculdade Eça de Queirós. 6(1): 2179-9636. http://uniesp.edu.br/sites/_biblioteca/revistas/20170509162633.pdf

Martins A. P. O. Q., Peres A. M., Gil N. L. M., Ros C., Lowen I. M. V. \& Gonçalves L. S. (2017). Usabilidade do Prontuário Eletrônico em Unidades Básicas de Saúde. Ciência, Cuidado e Saúde. Umuarama. 2017 Abr/Jun; 16(2). DOI: 10.4025/cienccuidsaude.v16i2.29748

Matsuda L. M., Évora Y. D. M., Higarashi I. H., Gabriel, C. S. \& Inoue, K. C. (2015). Informática em enfermagem: Desvelando o uso do computador pelos enfermeiros. Texto contexto - enferm. Florianópolis. 24(1): 178-86. https://www.scielo.br/pdf/tce/v24n1/pt_0104-0707-tce-24-01-00178.pdf.

Medeiros J. B., Holmes E. S., Albuquerque S. G. E., Candeia R. M. S. \& Costa T. (2017). O e-SUS Atenção Básica e a Coleta de Dados Simplificada: Relatos da implementação em uma Estratégia Saúde da Família. Rev. APS. 20(1): 145-9. https://doi.org/10.34019/1809-8363.2017.v20.15784

Mieiro D. B., Oliveira E. B. C., Fonseca R. E. P., Miniel V. A., Zem- Mascarenhas, S. H. \& Machado, R. C. (2019). Estratégias para minimizar erros de medicação em unidades de emergência: Revisão Integrativa. Rev Bras Enferm. Brasília. 72(1): 320-7. https://doi.org/10.1590/0034-7167-2017-0658

Ministério da Saúde (BR) (2020). Departamento de Informática do SUS (DATASUS). SI-PNI Sistema de Informação do Programa Nacional de Imunização. DATASUS. http://pni.datasus.gov.br/

Ministério da Saúde (BR) (2016). Diretoria de Vigilância Epidemiológica Estado de Santa Catarina, Superintendência de Vigilância em Saúde. Plano de ampliação de testes rápidos nos serviços de saúde de Santa Catarina. https://www.saude.sc.gov.br/index.php/documentos/legislacao-principal/anexos-dedeliberacoes-cib/anexo-deliberacoes-2016/10961-anexo-deliberacao-238-2016-plano-estadual-ampliacao-teste-rapido-2016/file.

Ministério da Saúde (BR) (2013). Instituto Nacional do Câncer José Alencar Gomes da Silva (Inca). Sistema de Informação do Câncer: manual preliminar para apoio a implantação. Rio de Janeiro. https://www.saude.sc.gov.br/index.php/documentos/atencao-basica/saude-da-mulher/siscan/7171-manual-preliminarsiscan/file.

Pissaia L.F., Costa A. E. K., Moreschi C. \& Rampel C. (2016). Sistematização da assistência de enfermagem: impacto da informática e os desafios na qualidade da assistência. Saúde.com. 12(4): 737-43. DOI 10.22481/rsc.v12i4.483

Portela G. Z. (2017). Atenção Primária à Saúde: um ensaio sobre conceitos aplicados aos estudos nacionais. Physis, Rio de Janeiro. 27(2): 255-76. https://doi.org/10.1590/s0103-73312017000200005.

Santos T. O., Pereira L. P. \& Silveira D.T. (2017). Implantação de sistemas informatizados na saúde: uma revisão sistemática. Revista Eletrônica de Comunicação, Informação e Inovação em Saúde. 11(3): 1-11. https://doi.org/10.29397/reciis.v11i3.1064

Cavalcante R. B., Silva T. I. M., Silva H. R. M. Santos R.C., Guimarães E. A. A. \& Pinheiro M. M. K. (2018) Difusão da Inovação Tecnológica e- SUS AB: aceitação ou rejeição? Cogitare enferm. 3(23): e55911. http://dx.doi.org/10.5380/ce.v23i3.55911

Werle G. \& Medeiros C. R. G. (2017). Os desafios do Enfermeiro na coordenação da Unidade Básica de Saúde. Artigo (Graduação) - Curso de Enfermagem, Universidade do Vale do Taquari - Univates, Lajeado. Disponível em: https://univates.br/bdu/handle/10737/1981?mode=full. 